\title{
Towards Clinically Available Closed-loop Systems for Mechanical Ventilation in the Intensive Care Unit
}

\author{
M. Tschopp ${ }^{1,2}$, C. Lovis' \\ ${ }^{1}$ Intensive Care Unit, Geneva University Hospitals, Geneva, Switzerland \\ ${ }^{2}$ Service of Medical Informatics, Geneva University Hospitals, Geneva, Switzerland
}

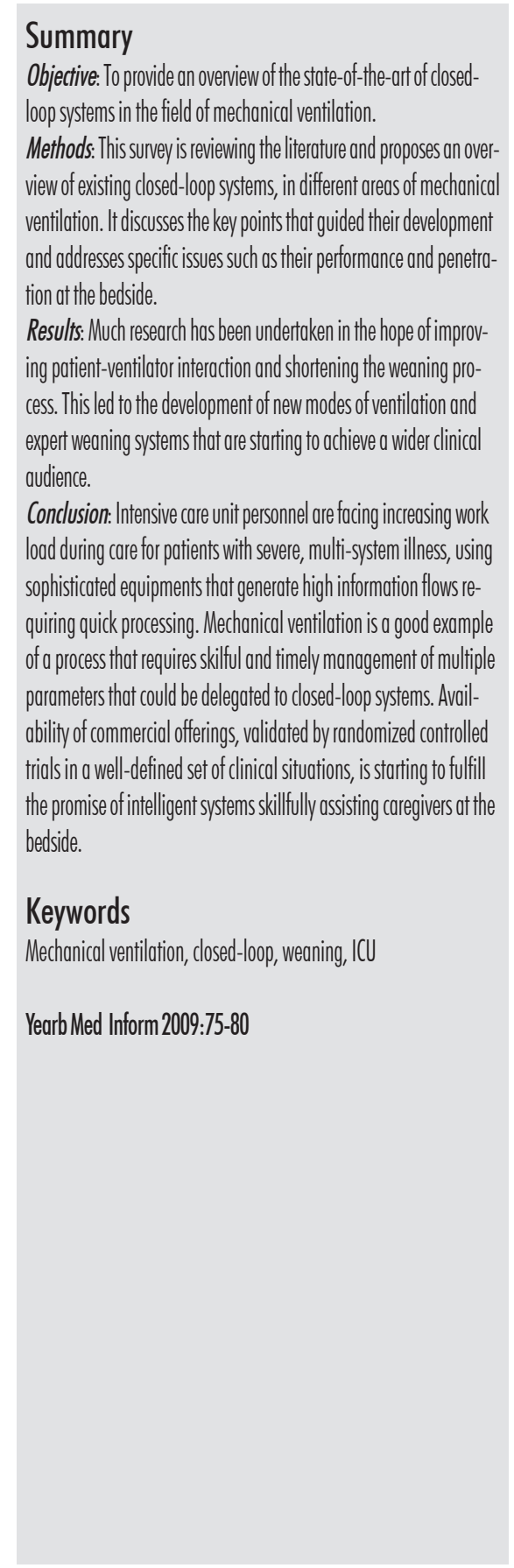

\section{Introduction}

Many factors contribute to increase the work and cognitive load of doctors and nurses working in intensive care units (ICUs). ICU personnel have to look after a progressively more ill and aging population [1]. They use sophisticated monitoring devices generating an evergrowing flow of information [2]. They have to master complicated equipment and engage up-to-date therapies at the bedside. They are compelled by the medical literature to enforce a growing set of guidelines [3], advocating tighter control over various clinical variables and processes [4]. Lastly they are under pressure from hospital cost-containing policies and worsening nursing shortage casting a shadow over the prospect to maintain, let alone increase, existing nurse-to-patient ratios [5].

In this setting, one can easily understand that there has been a strong impetus for development of intelligent solutions that would assist ICU workers, either by taking care of time-consuming and repetitive tasks, intuitively felt to be more accessible to automated systems, or by helping in situations where quick processing of large amounts of data could improve decision-making [6].

The fundamental characteristics of the ICU - a closely monitored and very technical environment, with an historic focus on research and often at the forefront of technological advances [7]made it a fertile ground for development and implementation of closedloop systems, aimed at regulating and influencing various physiopathological processes.
Mechanical ventilation is a good example of a technique that requires tight monitoring and frequent interventions from skilled personnel, in order to minimize complications and optimize outcome. A lot of research has been undertaken in the last decade to build intelligent systems that would be able to independently address many aspects of mechanical ventilation, most notably patient-ventilator interactions and weaning.

\section{Methods}

This article aims to give an overview on the state-of-the-art of closed-loop systems in the field of mechanical ventilation. It reviews the recent literature on the subject and gives a quick overview of the different systems that have been developed and tried, with a focus on commercially available solutions. Evidence, as gathered in clinical trials, is also looked at.

\section{Results}

\section{Basic Principles of Closed-loop Controls in Medicine}

Control theory has its roots in engineering and mathematics and studies the behavior of and control of dynamic systems. The basic principles of control theory state that when the desired output of a system needs to be maintained within a reference value, the controller adjusts the system input thru a feedback loop to obtain the desired 
effect on the output. The nature of the controller defines the way the input is going to be adjusted, based on the difference between the actual output and the desired set point [8]. Such systems clearly have applications in medicine, where homeostasis of physiological processes is important and accessible to simple feedback loops. Technical challenges in implementing such solutions in a clinical setting include:

- Selection of an output variable that is easily accessible to continuous monitoring.

- Design of an effective controller that allows quick and reliable correction of errors between the desired and observed output.

- Meeting stringent quality and safety standards

\section{Background}

Classically, the ventilatory support delivered to mechanically ventilated patients is manually adjusted by doctors, nurses or physiotherapists [9]. This paradigm has somehow changed in recent years, as ventilators started to incorporate new ventilation modes and sophisticated functions based on the principles of closed-loop controls. While research on such technology predates the era of microprocessor-based ventilators [10], technological advances in recent commercial offerings are now making those concepts accessible to a growing number of clinicians [11]. Closed-loop systems were historically developed along three intersecting axes: 1) maintenance of homeostasis of the respiratory system, 2) improvement of patient-ventilator interaction and 3) reduction of the duration of weaning. Systems reviewed in this article will be organized and discussed under those three broad categories.

\section{Maintaining Homeostasis}

Mechanical ventilation is classically administered to patients with an inabil- ity to maintain proper oxygenation and ventilation. Those two dimensions are easily monitored continuously and noninvasively by pulse oximetry and endtidal $\mathrm{CO} 2$ (EtCO2) monitoring respectively. Furthermore, there is a clear, if not single and immutable, physiologic relation between them and ventilator controlled or observed variables, such as tidal volume $(\mathrm{Vt})$, respiratory rate (RR) and fractional inspired concentration of oxygen (FiO2). This observation made homeostasis of oxygen and carbon dioxide one of the first fields of research in closed-loop ventilation. Fig. 1 illustrates such a simple model.

Although all the systems described in this article are designed to maintain the homeostasis of one or more physiological variables, and could as such be listed under the current category, the following two examples were chosen because they focus exclusively on a single input and output.

\subsection{Carbon Dioxide Homeostasis}

Seminal work published by Saxton and Myers [10] in 1956 paved the way for closed-loop developments in the field of mechanical ventilation. He described a servomechanism for automatic regu- lation of ventilation in patients ventilated by negative-pressure ventilators (known as "Tank respirators" or "Iron lungs"). The output observed variable was an expired $\mathrm{CO} 2$ infrared analyzer whose readings were compared to normal preset values. High and low levels of $\mathrm{CO} 2$ were corrected by operating the respirator thru a servomechanism that opened or closed the valve controlling the level of pressure in the tank. An unknown number of patients were treated using this device but at least two achieved successful CO2 homeostasis for a limited period of time.

\subsection{Oxygenation Homeostasis}

More recent work, focusing on the oxygenation component, was published by Tehrani [12] in 2003. It describes a closed-loop system designed for automatic adjustment of FiO2, based on analysis of pulse oximetry. The originality of this work compared to earlier attempts, is that it incorporates two different control mechanisms. One responds quickly to fast declines of oxygen saturation ( $\mathrm{SpO} 2)$, with a big increment in $\mathrm{FiO} 2$, to prevent life-threatening hypoxemia, the other is a slower, classic proportional-integral-derivative

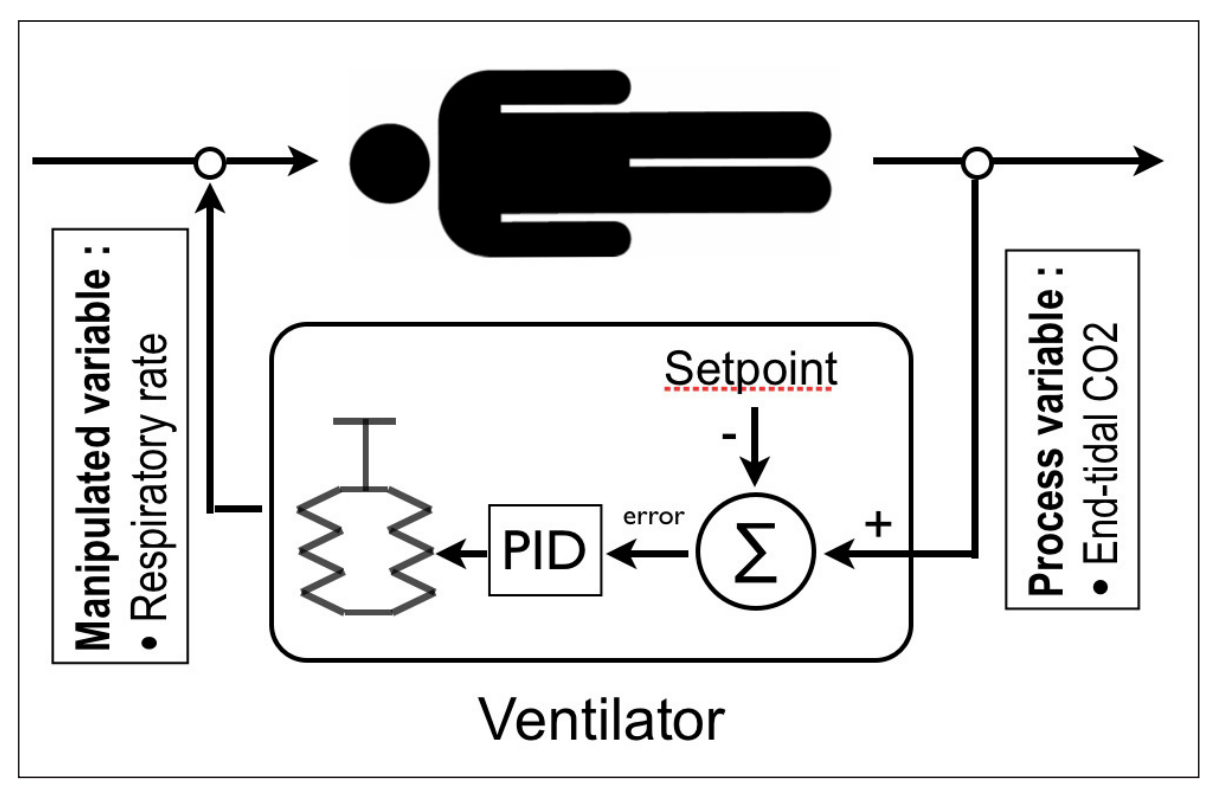

Fig. 1 Model of a simple PID closed-loop system with a positive feedback loop increasing the respiratory rate based on the patient's exhaled CO2 
(PID) control procedure, which allows fine-tuning of $\mathrm{FiO} 2$ when $\mathrm{SpO} 2$ is in a safe, normal range. Although never validated in humans in this particular form, it has now been integrated as part of a more advanced development [13], discussed later in this article.

\section{Improving Patient-ventilator Interaction (Specific Modes)}

Optimal patient-ventilator interaction is of great importance to achieve the two main goals of mechanical ventilation: adequate oxygenation and ventilation and reduction of respiratory muscle fatigue thru decreased work of breathing [14]. Over the years, much effort has been invested in the development of new modes of ventilation that held the promise of improving patient-ventilator synchrony, at the price of much confusion from clinicians, often feeling unable to master complicated settings or adequately choose between competing offerings [15]. Closed-loop systems, with their ability to monitor and adjust in real-time, without human interaction, the different variables of mechanical ventilation have been seen by many as a magic bullet and placed at the core of the following commercially-available ventilatory modes.

\subsection{Dual-Control (Adaptative Pressure Control)}

Adaptative Pressure Control (APC) is an enhancement of volume-controlled ventilation where the pressure-limit is not constant, but varies from one breath to the next, thanks to a closed-loop system that controls the output of the current breath based on a comparison of the targeted (set) and actual (delivered) tidal volume of the previous breath. The traditional constant flow of volume-controlled ventilation is replaced (after a test breath used to calculate the compliance of the respiratory system) with the variable flow waveform one would expect from pressure control modes, as the algorithm constantly adjusts the pressure limit to fit the preset tidal volume, while reducing peak inspiratory pressure (PIP). This bestof-both-world approach aims to provide a constant $\mathrm{Vt}$, immune to changes in compliance, while minimizing peak airway pressure. APC is implemented in most recent ventilators under different names (AutoFlow on the Dräger Evita series, Adaptative Pressure Ventilation on Hamilton's Galileo and Pressure Regulated Volume Control on the Servo-i by Maquette). The literature with regard to the benefit of APC is lacking but a recent review by Branson on small trials and case series failed to show any benefit beyond the expected reduction in PIP [16].

\subsection{Adaptative Support Ventilation}

Adaptative Support Ventilation's (ASV) paternity is a contentious subject [17, 18] but it is probably safe to say that its principles were defined in the early nineties by Tehrani and Laubscher [19], on the proposition to adjust $\mathrm{Vt}$ and $\mathrm{RR}$ to achieve minimal work of breathing, based on a model derived from pioneering work published by Otis in 1950 [20]. The algorithm does a breath-to-breath analysis of respiratory mechanics, by analysis of the expiratory flow-volume curve. Based on the desired minute ventilation set by the clinician, it continuously adapts the RR - Vt combination through changes in the level of inspiratory pressure and mandatory respiratory rate, to ensure minimal work on the part of respiratory muscles at the desired minute ventilation. This work was eventually licensed by Hamilton Medical and marketed as a distinct ventilatory mode on their line of ventilators. It is validated as an effective way of improving patient-ventilator interactions and decreasing respiratory muscle load and deemed as safe as conventional ventilation $[21,22]$.

\subsection{Proportional Assist Ventilation}

Proportional assist ventilation was developed in the early nineties by Younes
[23] and is a form of pressure-support ventilation that continuously adapts the level of inspiratory pressure according to a model using the equation of motion, which states that the pressure applied by respiratory muscles to the system is used to overcome elastic and resistive forces. Knowledge of normal elastance (E) and resistance (R) of the patient, as inputted by the clinician, and constant monitoring of the rate and volume of gas flow coming from the ventilator, allows the algorithm to calculate and deliver the correct amount of pressure support to compensate for increased elastic and resistive work of breathing. In summary, the higher the respiratory drive it observes from the patient, the more pressure (thus assistance) the machine generates. PAV is commercially available on respirators from Puritan Bennett and under the name of Proportional Pressure Support on the Dräger Evita Series. Clinical studies show better patient tolerance and comfort compared with conventional Pressure Support, but failed to show improved outcome [24]. Because the need for regular assessment of the patient's respiratory mechanics ( $\mathrm{R}$ and E) was deemed a major obstacle to the widespread use of this mode, an update labeled PAV + has been proposed which implements a method for automatic and non-invasive monitoring of $\mathrm{R}$ and $\mathrm{E}$ [25].

\subsection{Mandatory Minute Ventilation}

Mandatory Minute Ventilation was described in 1977 by Hewlett to assist weaning from mechanical ventilation [26]. In this mode the mandatory breath rate is continuously adapted to variations of the patient's spontaneous respiratory rate to meet predetermined minute ventilation (MV). If the patient's spontaneous breathing meets the preset MV, no mandatory breath will be administered. MMV is implemented on the Dräger Evita series and has been shown in a small randomized controlled trial to significantly reduce weaning time [27]. 


\section{Reducing Duration of Weaning}

The weaning process accounts for a significant part of the time a patient spends on mechanical ventilation [28] and failure to achieve separation from the ventilator in a timely fashion is strongly related to increased morbidity and mortality [29]. In deciding when time has come to extubate, clinical judgment alone is not perfect and tends to prolong the duration of ventilation [30]. Studies have shown that using a strict and systematic approach to weaning can reduce the duration of mechanical ventilation [31], but compliance in clinical practice is low [32]. In this setting, automated weaning solutions are believed to hold the key to improving the situation by partly taking the weaning process out of the hands of caregivers, thus addressing issues such as standard compliance, lack of resource and harmful variability in management.

\subsection{Knowledge-based Systems}

Knowledge-based systems were developed to overcome the following limitations of classic control systems, based on physiologic models [13]:

- Creating realistic and versatile models, valid for the variety of patients ventilated in an ICU, is a daunting and probably impossible task.

- Once designed, those models have to be fed with many parameters that may not be easily available to continuous monitoring at the bedside.

- Furthermore, having a model is not sufficient to drive a dynamic clinical process such as weaning, which extends over a period of time that goes well beyond the immediacy of a standard control loop. Control, the task of determining what to do the next instant, needs to be paired with a planning component, that will make strategic decisions and drive the long term process.

The originality of the work on knowledge-based systems is that it focuses on the modeling of the medical expertise required to address a clinical problem, rather than on comprehensive physiological models. The rationale behind this shift of focus is that, although the physiology of patients suffering from diverse conditions may be variable and complex, the decision making process of medical experts dealing with a specific, well defined problem, such as weaning, may be less variable, thus easier to model. Knowledge-based systems are thus well suited to drive strategic decisions in the complex process of weaning a patient from mechanical ventilation.

\subsection{NéoGanesh - SmartCare ${ }^{\mathrm{TM}}$}

A knowledge-based system for weaning of mechanical ventilation was described by Dojat in 1992 [33,34] and evolved in a solution integrated as a commercial component of the Dräger Evita XL series. It works by adapting the level of pressure support to the patient's ventilatory needs by continuously recording and analyzing the respiratory rate, the tidal volume and the end-tidal CO2 levels. The goal of the system is to keep the patient in a socalled comfort zone, in a defined range of the three monitored parameters. The level of support is periodically reduced to a minimal value where separation from the ventilator could be envisaged by the clinician. NéoGanesh is based on current artificial intelligence technologies and relies on the representation of the medical expertise acquired over a decade in a large ICU. NéoGanesh has been used to ventilate a large number of patients and has showed in controlled trials that it could perform as well, or better, than conventional management [35].

\subsection{Flex}

More recently another knowledge-based weaning system [13] has been described by Tehrani, expanding on earlier work on closed loop control of ventilation and oxygenation by the same author [12], notably Adaptative Support Ven- tilation and automatic titration of fractional inspired oxygen. It also incorporates principles of Mandatory Minute Ventilation development. Its goals are more ambitious than NéoGanesh because it can be used in a variety of ventilatory modes, is not restricted to weaning scenarios, and can provide closed-loop ventilation or behave as an advisory tool. It currently lacks clinical validation from randomized controlled trials.

\section{Discussion}

It has always been a feature of mechanical ventilation that the offerings in terms of ventilation modes and special functionalities weren't necessarily matched by a perceived need by the clinicians [11]. Mechanical ventilation is a difficult technique to master and people tend to use the one or two modes they have been trained on and are comfortable with. One can easily understand that new techniques face an uphill battle for the mind share of clinicians, especially when they involve shifts in paradigm, such as leaving the human out of the loop.

In a review of ventilator practice at the bedside [32], Rubenfeld states that ICUs have an historic inclination towards variable clinical practices, low observance of guidelines and overall resistance to change in practices. The lack of a rich evidence base of positive clinical trials, and the abundance of conflicting results in successive trials, allow intensivists to justify therapeutic decisions on a mixture of personal experience, physiologic rationale and personal values. Current evidence on closed-loop doesn't provide a strongenough body of evidence to induce a large change in behaviors.

Another element to consider, that is specific to closed-loop systems, is the black box effect. The lack of visibility of what the ventilator is actually doing, or how it is actually making its decisions, can induce an initial reac- 
tion of distrust. Working towards more descriptive displays and alert systems on ventilators could be the key to better acceptance of intelligent systems by medical and nursing teams [36].

Future development will undoubtedly focus on improving the intelligence the system has on the patient immediate status and predicted outcome. Large amounts of data are generated, and for the most part ignored, during the days a patient spends on mechanical ventilation. It's not unreasonable to try and apply data mining techniques to drive sophisticated alarms and display indicators on the patient's respiratory condition. Visualization of complex processes in a human-readable way, combining information from multiple sources, is also an area where interesting work is already taking place [37]. All those topics extend beyond the domain of mechanical ventilation in the ICU and will help push more general medical informatics concepts and solutions into the hands of carers at the bedside.

\section{Conclusion}

The rationale behind the use of closedloop ventilation is sound. There is an irresistible push to increase safety, enforce clinical guidelines, decrease unnecessary variability in processes of care, better use scarce nursing resources and take better advantage of the increasing stream of information that advanced monitoring devices deliver in real time. Closed-loop systems, in ventilation and hopefully in the near future in other domains, such as blood glucose management, hold the key to better address those pressing issues. Availability in a variety of commercial offerings is bridging the gap from the lab to the bedside. Randomized controlled trials have validated those techniques in a well-defined set of clinical situations. Now could be the time to enjoy the long overdue promise of automated systems, freeing valuable clinician's time that will be better invested on areas currently out of reach of automated systems.

\section{Glossary}

EtC02: end-tidal carbon dioxide. Noninvasive and continuous measurement used as a surrogate of the arterial partial pressure of $\mathrm{CO} 2$.

FiO2: fraction of inspired oxygen. Amount of oxygen mixed with air supplied to the patient. FiO2 of $100 \%$ is pure oxygen.

MV: minute ventilation. Tidal volume multiplied by respiratory rate.

PID: proportional integral derivative controller. A generic control loop feedback mechanism widely used to correct the error between a measured variable and a desired setpoint.

PIP: peak inspiratory pressure. Maximum airway pressure generated by the ventilator to move air in the lungs.

RR: respiratory rate, as dictated by the ventilator, the patient or a combination of both.

Sp02: Saturation of oxygen in the arterial blood, measure non-invasively and continuously with pulse oximetry.

$\mathbf{V t}$ : tidal volume. Volume of air that goes in and out of the patient with each breath.

\section{References}

1. Sparkes DJ, Smith GB, Prytherch D. Intensive care requirements for an ageing population-a microcosm of problems facing the NHS? Clin Med 2004 MayJun;4(3):263-6.

2. Heldt T, Long B, Verghese GC, Szolovits P, Mark RG. Integrating data, models, and reasoning in critical care. Conference proceedings: Annual International Conference of the IEEE Engineering in Medicine and Biology Society IEEE Engineering in Medicine and Biology Society Conference 2006;1:350-3.

3. Rolls KD, Elliott D. Using consensus methods to develop clinical practice guidelines for intensive care: the intensive care collaborative project. Aust Crit Care 2008;21(4):200-15.

4. van den Berghe G, Wouters P, Weekers F, Verwaest C, Bruyninckx F, Schetz M, et al. Intensive insulin therapy in the critically ill patients. N Engl J Med
2001;345(19):1359-67.

5. Odom J. Nursing shortage: impending doom or challenging opportunity? J Perianesth Nurs 2000;15(5):348-9.

6. Clifford GD, Long WJ, Moody GB, Szolovits P. Robust parameter extraction for decision support using multimodal intensive care data. Philosophical transactions Series A, Mathematical, physical, and engineering sciences 2009;367(1887):411-29.

7. Clemmer TP. Computers in the ICU: where we started and where we are now. J Crit Care 2004; 19(4):201-7.

8. Fields AM, Fields KM, Cannon JW. Closed-loop systems for drug delivery. Curr Opin Anaesthesiol 2008;21(4):446-51.

9. Greenwald I, Rosonoke S. Mechanical ventilation: understanding respiratory physiology \& the basics of ventilator management. JEMS 2003;28(12):74-86.

10. Saxton GA, Myers G. A servomechanism for automatic regulation of pulmonary ventilation. J Appl Physiol 1957;11(2):326-8.

11. Burns SM. Pressure modes of mechanical ventilation: the good, the bad, and the ugly. AACN Adv Crit Care 2008;19(4):399-411.

12. Tehrani F, Rogers M, Lo T, Malinowski T, Afuwape $\mathrm{S}$, Lum M, et al. Closed-loop control if the inspired fraction of oxygen in mechanical ventilation. J Clin Monit Comput 2002;17(6):367-76.

13. Tehrani F, Roum J. Flex: A New Computerized System for Mechanical Ventilation. J Clin Monit Comput 2008;22(2):121-30.

14. Prinianakis G, Kondili E, Georgopoulos D. Patient-ventilator interaction: an overview. Respir Care Clin N Am 2005;11(2):201-24.

15. Chatburn RL. Classification of ventilator modes: update and proposal for implementation. Respir Care 2007;52(3):301-23.

16. Branson RD, Chatburn RL. Controversies in the critical care setting. Should adaptive pressure control modes be utilized for virtually all patients receiving mechanical ventilation? Respir Care 2007;52(4):478-88.

17. Tehrani F Adaptive support ventilation is a patented technology Intensive Care Med 2008; 34(12):2306.

18. Wysocki M, Arnal J Response to dr. Tehrani. Intensive Care Med 2008;34:2307-8.

19. Laubscher TP, Heinrichs W, Weiler N, Hartmann G, Brunner JX. An adaptive lung ventilation controller. IEEE Trans Biomed Eng 1994;41(1):51-9.

20. Otis AB, Fenn WO, Rahn H. Mechanics of breathing in man. J Appl Physiol 1950; 2(11):592-607.

21. Sulzer CF, Chioléro R, Chassot PG, Mueller XM, Revelly JP. Adaptive support ventilation for fast tracheal extubation after cardiac surgery: a randomized controlled study. Anesthesiology 2001; 95(6):1339-45.

22. Arnal JM, Wysocki M, Nafati C, Donati S, Granier I, Corno G, et al. Automatic selection of breathing pattern using adaptive support ventilation. Intensive Care Med 2008;34(1):75-81.

23. Younes M. Proportional assist ventilation, a new approach to ventilatory support. Theory. Am Rev Respir Dis 1992;145(1):114-20.

24. Ambrosino N. Proportional assist ventilation (PAV): a significant advance or a futile struggle between 
logic and practice?.Thorax 2002:57(3):272-6.

25. Younes M, Kun J, Masiowski B, Webster K, Roberts D. A method for noninvasive determination of inspiratory resistance during proportional assist ventilation. Am J Respir Crit Care Med 2001; 163(4):829-39.

26. Hewlett AM, PlattAS, Terry VG. Mandatory minute volume. A new concept in weaning from mechanical ventilation. Anaesthesia 1977 Feb;32(2):163-9.

27. Davis S, Potgieter PD, Linton DM. Mandatory minute volume weaning in patients with pulmonary pathology. Anaesth Intensive Care 1989 May;17(2):170-4.

28. Esteban A, Alía I, Ibañez J, Benito S, Tobin MJ. Modes of mechanical ventilation and weaning. A national survey of Spanish hospitals. The Spanish Lung Failure Collaborative Group. Chest 1994; 106(4):1188-93

29. Torres A, Aznar R, Gatell JM, Jiménez P, González $\mathrm{J}$, Ferrer A et al. Incidence, risk, and prognosis factors of nosocomial pneumonia in mechanically ventilated patients. Am Rev Respir Dis 1990; 142(3):523-8
30. Ely EW, Baker AM, Dunagan DP, Burke HL, Smith $\mathrm{AC}$, Kelly PT et al. Effect on the duration of mechanical ventilation of identifying patients capable of breathing spontaneously. N Engl J Med 1996; 335(25):1864-9.

31. Lellouche F, Mancebo J, Jolliet P, Roeseler J, Schortgen F, Dojat M et al. A multicenter randomized trial of computer-driven protocolized weaning from mechanical ventilation. Am J Respir Crit Care Med 2006;174(8):894-900.

32. Rubenfeld GD. Implementing effective ventilator practice at the bedside. Curr Opin Crit Care 2004; 10(1):33-9.

33. Dojat M, Brochard L, Lemaire F, Harf A. A knowledge-based system for assisted ventilation of patients in intensive care units. Int J Clin Monit Comput 1992;9(4):239-50.

34. Dojat M, Pachet F, Guessoum Z, Touchard D, Harf A, Brochard L. NéoGanesh: a working system for the automated control of assisted ventilation in ICUs. Artif Intell Med 1997;11(2):97-117.

35. Lellouche F, Mancebo J, Jolliet P, Roeseler J, Schortgen F, Dojat M et al. A multicenter random- ized trial of computer-driven protocolized weaning from mechanical ventilation. Am J Respir Crit Care Med 2006;174(8):894-900.

36. Wysocki M, Brunner J. Closed-Loop Ventilation An Emerging Standard of Care? Crit Care Clinics 2007;23(2):223-40.

37. Wachter SB, Johnson K, Albert R, Syroid N, Drews F, Westenskow D. The evaluation of a pulmonary display to detect adverse respiratory events using high resolution human simulator. J Am Med Inform Assoc 2006; 13(6):635-42.

\section{Correspondence to:}

Mathius Tschopp, MD

Geneva University Hospital

Rue Gabrielle-Perrete-Gentil 4

CH-1211 Geneva 14

Switzerland

Tel : +41 223726180

Fax: +41223728680

E-mail: mathias.tschopp@sim.hcuge.ch 